\title{
La tecnicalità è un (prestito di) lusso?
}

\author{
Matilde Paoli
}

PUBBLICATO: 09 APRILE 2019

\section{Quesito:}

Da qualche tempo, così ci segnalano anche alcuni lettori, nel linguaggio riferibile agli ambiti aziendale, politico e giornalistico si impiega il termine tecnicalità. Questa voce sembra suscitare una certa diffidenza: una lingua che annovera nel suo vocabolario tecnica, tecnicismo, tecnicitá, tecnicizzazione, tecnificazione, tecnologia, per citare solo i sostantivi, ha davvero bisogno di una parola nuova, per di più importata dall'inglese?

\section{La tecnicalità è un (prestito di) lusso?}

$\mathrm{E}$ ffettivamente tecnicalità è adattamento dell'inglese technicality, voce risalente alla seconda metà del Settecento, a sua volta derivata dall'aggettivo technical (databile all'inizio del XVII secolo) che ha per base il latino tardo technicus o forse direttamente il greco technikós (cfr. OED s.v.).

Che sia una parola "dell'ultima ora" è più difficile da sostenere, visto che alcuni dizionari l'attestano da tempo: lo Zingarelli lo fa dal 1996 ed era già presente in Nuove parole italiane dell'uso (2003) supplemento all'edizione 2000 del GRADIT; la troviamo poi nel dizionario Hoepli zor e nel Garzanti 2013, nel Treccani 2014 e nel Vocabolario Treccani online. Prima dello Zingarelli la registrava il repertorio di neologismi Il dizionario italiano: parole nuove della seconda e terza repubblica di Silverio Novelli e Gabriella Urbani (Roma, Datanews, 1995).

Per quel che riguarda la datazione, Zingarelli fa risalire il suo ingresso in italiano al I987, GRADIT al I99I mentre in Novelli-Urbani si citano due passi da "Repubblica" entrambi del I994.

Sul significato c'è maggiore uniformità: "espediente, dettaglio tecnico" (Novelli-Urbani I995); "dettaglio tecnico: non perdete tempo in inutili tecnicalita" (Zingarelli 1996); "spec. con connotazione negativa, particolare, dettaglio tecnico" (GRADIT); "Aspetto specificamente e aridamente tecnico; dettaglio tecnico" (Hoepli 20rI); "non com. - Piccolo dettaglio altamente tecnico di un problema, poco comprensibile ai non esperti" (Vocabolario Treccani online e Treccani 20I4). Solo Garzanti 20I3 ha un valore diverso: "eccessiva attenzione verso i dettagli tecnici di norme, regolamenti, atti amministrativi, burocratici ecc.". Si noti che in quasi tutte le descrizioni c'è una più o meno velata connotazione negativa: esplicita in GRADIT e implicita nelle descrizioni o negli esempi negli altri dizionari.

Trattandosi di derivazione dall'inglese vediamo cosa si intenda con technicality in quella lingua (definizioni tratte dall'OED, traduzione nostra):

r.a. A technical issue, detail, or term belonging to a particular field. Usually in plural ['un problema, un dettaglio tecnico o un termine appartenente a uno specifico ambito. Solitamente al plurale'].

r.b. A point or a detail of a set of rules; spec. a minor legal point, esp. when considered as trivial or when used to evade the intention of the law. Also in extended use ['un aspetto o un dettaglio di un insieme di regole; in senso specialistico un aspetto legale minore, specialmente quando considerato banale o quando usato per eludere la legge. Anche in usi figurati']. 
2. The state or quality of being technical; the use of technical terms or methods ["lo stato o la qualita di ciò che è tecnico; l'uso di termini o metodologie tecniche'].

Di questi valori alcuni hanno già un puntuale corrispettivo italiano: tecnicismo vale "parola o locuzione che fa parte di un linguaggio tecnico' (GRADIT) corrispondente quindi al secondo valore in Ia. La forma italiana che esprime quanto espresso in ib. è cavillo (legale) che ne condivide anche la notazione in senso negativo, mentre per la prima definizione riportata in 2. la nostra lingua dispone di tecnicitá. Per il secondo senso riportato in 2. potrebbe essere usato forse ancora tecnicismo che però ha accezione spregiativa e decisamente più circoscritta: "spreg. uso eccessivo di termini tecnici nel parlare e nello scrivere" (GRADIT). Resta comunque scoperto (a meno di non usare il sintagma dettaglio/aspetto tecnico) il valore primo di ra. che è appunto quello assegnato dalla lessicografia a tecnicalità.

Veniamo all'uso: consultando il corpus di Google Libri la prima attestazione ci risulta nel New english and italian pronouncing and explanatory dictionary di John Millhouse (Milano, i8704) in cui la voce Téchnicálity è tradotta con tecnicalitá. Ma questo non ci dice niente sul reale impiego né sul valore del termine.

L'anno successivo troviamo un'attestazione sul settimanale "Gazzetta Biellese" (n. 2o, anno VIII, I7/05/I87I, p. 3), in un testo che promuove Corsi di lingua inglese e spagnuola tenuti dal "Prof. Manetta, Segret. Onor. della Società antropologica di Londra, e professore patentato della R. Università di Torino". Il testo si conclude con queste parole:

[il professore] si rende garante che dopo sessanta lezioni i suoi allievi potranno leggere speditamente e tradurre colla massima facilità dall'inglese nella propria lingua. Oltre di ciò egli s'impegna di insegnare ai suoi allievi lo stile e le tecnicalità commerciali.

Il testo è firmato con una X, ma è forse possibile ipotizzare, anche in base ad altri elementi che non riportiamo, che l'autore (o almeno l'ispiratore) sia lo stesso professor Manetta il quale aveva sicuramente presente le Commercial Technicalities, cioè i tecnicismi commerciali.

Successivamente appare nella rivista "L'omiopatia [sic] in Italia" (Unione tipografico-editrice, anno I9II, p. 34): “...scrivere materia d'attualità in modo popolare, libera da tecnicalità, vagliata, piana e facile." Nel passo si può riconoscere il valore di "termine o dettaglio tecnico (quindi di non facile comprensione)' della forma inglese.

Dopo qualche decennio, nel I958, lo ritroviamo ancora in una rivista, la "Rivista di economia agraria", in un brano, probabilmente una traduzione dall'inglese, che tratta di politica economica. Anche in questo caso ci si riferisce a tecnicismi da evitare per ottenere una scrittura piana, tesa alla divulgazione.

Le attestazioni successive risalgono agli anni Settanta: nel decennio se ne trovano otto o, meglio, sette visto che un passo si ripete identico in due pubblicazioni diverse. In tre casi la forma è al plurale: in due si parla di tecnicalitá giuridiche, mentre in un caso si fa riferimento all'insegnamento del latino. Riportiamo questo passo perché vi troviamo esplicitato cosa si intenda per tecnicalitá:

Per Terenzio quest'anno sopprimerò le tecnicalità, non farò cose mostruose come sillaba ancipite e via; voglio farvi sentire la latinità viva e mortale. Voi sapete bene che i Romani non dicevano ogni giorno «usque tandem, Catilina...», ma parlavano come uomini comuni (Dalle citazioni di Eduard Fraenkel sull'Eunuco, "Belfagor", vol. XXV, i97o, p. 673 [la citazione è datata Ir/4/1969]). 
Uno degli usi al singolare si riferisce ancora a Eduard Fraenkel del quale si cita l'"empirismo nemico di ogni rigida pastoia metodologica e di ogni tecnicismo eccessivo (o 'tecnicalità', come diceva con anglismo caratteristico)" (Luigi Enrico Rossi, premessa a Due seminari romani di Eduard Fraenkel: Aiace e Filottete di Sofocle, "Sussidi eruditi", vol. XXVIII, I977, p. XVI). Gli altri trattano "della tecnicalità dell'impresa", di "tecnicalità monetaria" o di tecnicalità tout court.

Nello stesso decennio il termine si affaccia anche sulla stampa nazionale; troviamo due attestazioni nel I970 e una nel 977 sempre sul "Corriere della Sera" e sempre tra virgolette:

La decisione ha suscitato sorpresa, irritazione, in chi ritiene che le «tecnicalità» giuridiche non dovrebbero lasciare via libera alle manifestazioni del malcostume politico ([s. f.] La frana di Agrigento, 2I $/ 2 / 1970$ ).

...essi devono, vogliono credere nella sua efficacia [del siero], e hanno fretta, contendono il passo alla morte, non capiscono le «tecnicalità» degli scienziati-burocrati (Giuseppe Josca, Bonifacio atteso a Milano, 2I/2/1970).

...al di là delle «tecnicalità»e delle differenze dottrinarie, sta diventando valore acquisito il riconoscimento dei diritti delle classi subalterne... (U[mberto] E[co], I comunisti fra antemarcia e cacciatori di streghe, $15 / 2 / 1977)$.

Ci pare che in questi passi si delinei una sorta di divaricazione tra tecnicalità (sempre al plurale che rimanda, come abbiamo visto, al valore di 'dettaglio, aspetto tecnico') da un lato, e, dall'altro, i grandi problemi della condizione umana, come nell'articolo di Josca, o la sostanza dei diritti, come nell'articolo di Eco.

Negli anni Ottanta troviamo otto attestazioni nel corpus di Google Libri che testimoniano l'uso del termine in politica (per fare un esempio: "la tecnicalità dello Stato liberal-borghese" è citata su "Panorama" Edizioni 783-788, I98I, p. III), in economia ("gli operatori non hanno ancora raggiunto lo spessore e la tecnicalità di operazioni similari effettuate nei mercati finanziari più sofisticati", Le Società IPSOA, I986, p. 43), nella lingua della burocrazia ("gestire lo «strumento» con la«tecnicalità»", Previdenza sociale 1989, Istituto nazionale della previdenza sociale, vol. 45, p. 468), perfino in poesia:

Però ci sono dei vuoti - i vestiti / Che avevi... Bruciali! / E poi / Le necessarietecnicalità / L'esatta successione Della parziale sia pur spoliazione Vuole il minuto vero - A cosa fosse intento Essere nell'inerme suo frattempo (Giovanni Giudici, Lume dei tuoi misteri 1984, p. 50).

Per quel che riguarda la stampa, negli anni Ottanta si contano so attestazioni, di cui la prima, del I984, in un articolo che tratta di politica economica apparso sulla "Stampa":

tutti, persino la Confcommercio, si affrettano a proclamare che i presupposti sono giusti per poi subito dopo aggiungere che bisogna emendarla [la legge Visentini sulle norme per la stesura dei bilanci societari], rivederla in questo o quel punto, perfezionarne il meccanismo. Ma dietro l'apparente «tecnicalità»vi è la speranza di ridurla ad una ragnatela più che a uno scudo (Mario Pirani, $I$ commercianti e il piano Visentini Saracinesca selvaggia, I4/Io/1984).

Anche in questo caso c'è una contrapposizione tra tecnicalità (al singolare quindi come 'argomentazione di carattere tecnico' o qualcosa del genere) e intenzione reale: la tecnicalità che ha (o dovrebbe avere) carattere neutrale, obiettivo, è utile a nascondere interessi di parte. 
Ancora sulla "Stampa" la voce compare nel 1986 in un articolo di ambito economico. Al I987 risalgono le prime attestazioni sulla "Repubblica", in due articoli che riportano le stesse parole di Mario Schimberni amministratore delegato della Montedison: "Il consiglio ha preso atto della situazione riservandosi di definire al momento opportuno modalità e tecnicalità compatibili con la situazione dei mercati" (Marco Panara, La Montedison cambia rotta salta l'aumento di capitale, II/II e Id. e Antonio Ramenghi, Schimberni Gardini atto secondo del I3/II). Su queste occorrenze si fonda probabilmente la datazione dello Zingarelli 1996.

Lo stesso quotidiano, a quel che ci risulta il primo a farlo tra quelli presi in esame, nel 1985 aveva introdotto la forma inglese:

... Ma il punto di sostanza è altro: quel che ci si aspettava da questa riforma era qualcosa di ben più ampio e profondo di una revisione delle "technicalities" finanziarie (Massimo Riva, Povero mezzogiorno, I3/4/1985).

Negli anni successivi abbiamo ancora apparizioni sporadiche circoscritte ad ambiti tecnici, soprattutto economia e finanza, spesso tra virgolette o in carattere corsivo. Sulla "Repubblica" continua a presentarsi anche il termine inglese. Riporto il passo relativo alla terza attestazione sulla "Repubblica", sezione Affari e finanza, che risale al I989, in cui l'autore fornisce esempi di ciò che considera tecnicalitá, ovvero modalità e procedure tecniche di formule di finanziamento e non solo dettagli come si legge in alcuni vocabolari. Si tratta quindi di un valore privo di qualsiasi connotazione negativa.

... sono poco innovative [le banche italiane], non offrono alla loro clientela, sia essa industriale o privata, quelle tecnicalità che sono ormai standard all'estero. Ad esempio, nel nostro paese i mutui per l'acquisto di un'abitazione difficilmente superano il 50 per cento del valore dell'immobile, mentre in Gran Bretagna le building societies possono arrivare anche al roo per cento, per di più senza eccessive formalità e abbinando il mutuo ad una polizza assicurativa sulla vita. E ancora, da noi troviamo formule di finanziamento all'industria o di ricoperture sui mercati dei cambi offerte soltanto presso le grandi banche o sulle principali piazze finanziarie, mentre nel resto del paese vigono sistemi arretrati (g. m., Lo straniero bussa allo sportello, "la Repubblica" 27/10/1989).

Gli anni Novanta vedono un notevole incremento dell'uso del termine sia sui libri (con l'intervallo di date impostato al decennio Google Libri mostra 25 copertine di testi di argomento sociologico, giuridico, economico e politico), sia, soprattutto, sulla stampa. "la Repubblica" si distingue con le sue 4I occorrenze, seguita dalla "Stampa" con 30 e dal "Corriere" che ne ha solo II. In questi stessi anni anche sulla "Stampa" e sul "Corriere" compaiono technicality e technicalities.

È questo il decennio in cui prima il repertorio di neologismi di Novelli e Urbani e poi la lessicografia registrano la voce. Ciò avviene anche grazie all'uso che ne fa un personaggio al centro dell'attenzione mediatica, Silvio Berlusconi, al quale viene spesso attribuita l'introduzione del termine in politica. In realtà sia in ambito imprenditoriale sia in ambito politico ci sono almeno due precedenti: Carlo De Benedetti in un'intervista ad Alberto Statera citata da Roberto Petrini (In ansia il 'BOT-people' Il congelamento fa 90 "la Repubblica" 2I/7/I99I) e Vasco Errani (Fatti e persone, "la Repubblica" I3/I2/I99I).

Berlusconi, stando ai corpora dei quotidiani esaminati, usa il termine per la prima volta parlando alla stampa nel 1993, come testimoniato in due articoli datati ${ }^{\circ}$ giugno nei quali si riportano le sue parole a proposito di Davide Giacalone, definito dal Cavaliere "uno dei più competenti per quanto riguarda la tecnicalità e la legislazione in materia di televisione" ([senza firma] Quel Giacalone riarrestato tanto stimato dalla Fininvest, "la Repubblica"; [gio. bia.], Berlusconi, faccia a faccia col giudice, "la Stampa"). Si noti che in questo caso il valore del termine, usato al singolare, non è dettaglio (insignificante), ma 
piuttosto 'linsieme degli aspetti tecnici riguardanti uno specifico settore (la cui comprensione richiede specifiche competenze)'.

In ogni caso è certamente in questi anni che l'attenzione si focalizza sulla parola:

Agli altri candidati il presidente risponde con la parola chiave «tecnicalità», che significa: «ai dettagli pensano i miei esperti». È questo il neologismo che si può mettere a insegna della complessità dello scontro cui abbiamo assistito (Ilvo Diamanti, Renato Mannheimer, Milano a Roma: guida all'Italia elettorale del 1994, Roma, Donzelli, 1994, p. 150).

Sul "Corriere della Sera" in un articolo di Giulio Nascimbeni, La nuova lingua da Sarchiapone a Internet del Io/II/rg95 che presenta la nuova edizione dello Zingarelli (lo Zingarelli ig96 appunto) tra le parole segnalate come "nuovi ingressi" si riporta tecnicalità specificandone il significato di 'dettaglio tecnico'. A chiusura di decennio e di secolo sulla "Repubblica" viene pubblicata a cura di Fabio Rossi ( $D a$ Berlusconia a Ribaltone, 7/9/1999) una lista alfabetica di "alcuni dei principali neologismi provenienti o propagati dai mezzi di comunicazione di massa dal 1990 ad oggi" dove alla lettera T tra teatrino della politica e teleasta troviamo il nostro tecnicalitá.

Negli stessi anni la parola, ormai adottata dalla politica, comincia a infiltrarsi in altri campi:

Il boato d'applausi che ha seguito l'altra sera il chiudersi del sipario sulla drammatica delusione che coglie Armida all'abbandono di Rinaldo, mentre il suo palazzo incantato veniva atterrato da un terremoto e dalle fiamme, non ha niente a che vedere, se non per via trasversa, con queste tecnicalità da storia della musica e del melodramma (Enzo Siciliano, Gluck, una fuga dal trash, "la Repubblica", 9/12/1996).

Con il XXI secolo continua il processo di affermazione e di espansione. Il corpus di Google Libri mostra 3.00o titoli: agli argomenti già visti si aggiungono l'arte, la comunicazione in rete, la filosofia, la pedagogia e la psicologia, in particolare la psicologia clinica:

Danni ancor maggiori, se fosse possibile, ha provocato la tecnicalità, vale a dire la centralità del potere orientato dalla sola competenza tecnica, nella produzione e nella progettazione di beni e di servizi. [...] Si pensi paradigmaticamente, alla sanità ed al prevalere della tecnicalità medica sulla domanda del paziente; paziente per lunghi anni privato di ogni "voce in capitolo", della stessa possibilità di esprimere le sue preoccupazioni, il suo parere, il suo punto di vista... (Renzo Carli, Rosa Maria Paniccia, L'analisi emozionale del testo, Franco Angeli, $2004\left(2002^{\mathrm{I}}\right)$ p. I2I).

In questo passo il termine indica 'l'insieme delle competenze, delle procedure tecniche', un valore quindi non negativo di per sé, ma solo se considerato esclusivo fondamento del rapporto con il paziente.

Sulla stampa, benché la politica resti l'ambito di affermazione del termine, ci imbattiamo ormai in tecnicalità di ogni tipo:

Il ministro della Sanità, Umberto Veronesi, propone realisticamente di combattere la diffusione dell'Aids con una «tecnicalità», l'utilizzo del preservativo, riducendone il prezzo [...] Sulla questione della droga sono a confronto due «tecnicalità» (il proibizionismo e l'antiproibizionismo) [...]. [...] (p[iero]. os[tellino] La tecnica e la morale, "Corriere della Sera" 2/I2/200o).

Alcune testimonianze riguardano la lingua delle aziende: all'interno del programma di un convegno pubblicizzato sulla "Stampa" del ı/9/2002 si prevede un intervento dal titolo "Uffici di oggi, uffici di domani: evoluzioni, tendenze e nuove tecnicalità per i manager immobiliari"; nelle offerte di lavoro 
pubblicate sullo stesso quotidiano il 3/5/2002 si legge che una "dinamica e qualificata società attiva nel campo della ricerca automobilistica avanzata" cerca un "exterior-interior designer in grado di presidiare le tecnicalità del ruolo esprimendo un forte spirito creativo innovativo...".

E ancora: sulla "Repubblica" il termine può essere usato anche parlando di scuola, o meglio, della riforma Moratti ("Come niente di speciale sono l'abolizione dell'esame di quinta elementare, le verifiche biennali e tante altre piccole tecnicalità...", Edmondo Berselli, La neo-scuola ideologica della Moratti, 2/2/2004), di guerre ("il generale Franks si racconta ma soprattutto spiega, o tenta di spiegare, la genesi e le tecnicalità di un conflitto che ha spaccato l'America e il mondo", Raffaella Menichini, Ingannai Saddam con una spia Il generale racconta la sua guerra, I/8/2004) e perfino di interventi chirurgici: "Quest' anno il ricavato verrà [...] destinato alla prevenzione e alla cura del cancro al seno, con l'impegno, [...], di approfondire le tecnicalità chirurgiche per la preservazione del capezzolo nel caso di mastectomia" (Francesca Floriani, Anna che va di corsa 'Cosi ho sconfitto il cancro', 24/4/2004).

Nel campo "privilegiato" della politica comincia a farsi largo un altro tipo di tecnicalità: quella elettorale.

...il premier prima di addentrarsi nella "tecnicalità" elettorale che doveva caratterizzare l'incontro, ieri mattina ha comunicato a tutti che la misura è colma: «Se qualcuno pensa di farmi bollire, posso assicurarvi una cosa: bollirà prima di me» (Augusto Minzolini, L'ira del premier: bollirete prima dì me "La Stampa" o7/02/2004 - numero 37 pagina 7).

In particolare nel 2005, in cui "Repubblica" registra un nuovo rialzo delle occorrenze che arrivano a 22, si intensifica l'uso del termine in rapporto alla nuova legge elettorale (L. n. 270 del 2I/I2), nota come legge Porcellum. In particolare lo usa cosi Berlusconi il quale parla del "sistema della tecnicalità del voto" (f.b., Berlusconi: Il partito unico? è fatto "la Repubblica" I6/6), di "una tecnicalità delle schede elettorali" (Berlusconi, alt alla successione Saró io il candidato nel 2006 "la Repubblica", 30/6), della "tecnicalità della legge elettorale" (Massimo Franco, La sfida sul premier puó riaprirsi in autunno, "Corriere della Sera", 3o/6).

Negli anni successivi si estendono ancora gli usi "trasversali" del termine: lo troviamo riferito all'enigmistica ("quell'impalpabile tecnicalità enigmistica...", Edmondo Berselli, Un popolo di cruciverbisti, "La Repubblica" I8/ir/2007), alla gestione di teatri, alle capacità teatrali ("Rocco Papaleo [..] esibisce una sicura tecnicalità di teatrante", Francesco Merlo, Rocco, il Monti del festival che rispecchia il Belpaese, la Repubblica I8/2/20I2), alla moda, alla scuola.

$\mathrm{Nel} 2015$ abbiamo un rilancio grazie a un altro protagonista politico, Matteo Renzi, che diviene il nuovo "uomo delle tecnicalità". Nelle 7 attestazioni relative a Renzi che troviamo sulla "Repubblica" si nota di nuovo la tendenza alla contrapposizione con la sostanza, con i "fondamentali" della politica. Riportiamo le più significative.

Sono i "fondamentali" del decreto e non cambieranno. "Il resto sono tecnicalità", garantisce Renzi (Goffredo De Marchis, L'ultimatum di Renzi: "LItalicum non cambia, non medio con Berlusconi", Repubblica.it, $9 / 2 / 2015)$.

"La tecnicalità di una riforma storica non è così rilevante da dedicarle due mesi di discussione..." ([senza firma], Riforma Senato, la maggioranza tiene. Grasso irritato: "No istituzioni come museo", Repubblica.it, $17 / 9 / 2015)$,

Gli anni 2016 e 2017 vedono in calo le occorrenze, ma in crescita gli ambiti di impiego: le tecnicalità si trasferiscono nel campo della tecnologia - per cui abbiamo tecnicalitá elettrotecniche e informatiche 
(Pier Luigi Bergondini, Emanuel e la moto: "Il mio casco intelligente puó salvare molte vite", la Repubblica Firenze.it, I4/3/20I7) e internettiane (Luciano Pilotti, Servizio civile per i migranti?, "Corriere della Sera", 30/9/2017), e in quello medico-sportivo.

Per lo scorso anno "la Repubblica" ha in attestazioni, mentre "la Stampa" e "il Corriere" ne contano rispettivamente 6 e Io. I giornalisti usano ormai il termine in riferimento il linguaggio tecnico o alle competenze tecniche di qualsiasi disciplina. Un esempio:

Ciò ha consentito loro [alle "nuove mafie"] di radicarsi in viluppi inestricabili di attività con altri malavitosi, imprenditori, amministratori, politici, servendosi di insospettabili "colletti bianchi" i quali, fungendo da cerniera tra mondo legale e illegale, mettono a disposizione le proprie tecnicalità, facilitano il riciclaggio, provvedono alla redazione di "speciali" contratti, pilotano appalti, forniscono ai criminali assistenza medica e legale (Luigi Labruna, Nuove mafie e colletti bianchi avanzano, "la Repubblica”, Iо/9/2018).

E naturalmente la usano i politici, anche quelli "del cambiamento":

Spiega [Giuseppe Conte] che non si andrà oltre il 2,04 per cento riformulato per il rapporto deficit-Pil: "Il saldo che abbiamo presentato è quello. È su quello che si possono costruire delle tecnicalità. Non abbiamo altri margini" ([s.f.], Manoura, Conte: "L'Italia non è con il cappello in mano. Trattativa a oltranza con l'Ue" Repubblica.it I4/ז2/2018).

Nel 2org (all'8/3) abbiamo solo una occorrenza sulla "Repubblica": "Il prezzo è la leva meno utilizzata perché spesso è considerata una tecnicalità" (Andrea Frollà, Prezzi, viaggi e software il puzzle di voli e crociere, "la Repubblica", 4/3/2019). Anche il prezzo quindi può essere una tecnicalità, ma forse ciò dipende dalle disponibilità di chi lo deve pagare.

In conclusione, si tratta di un termine presente nella nostra lingua da alcuni decenni, ma i periodi di frequenza decisamente bassa ne favoriscono a intervalli il rilancio come "novità" del momento, grazie anche all'uso da parte di personaggi famosi. Non è comunque voce molto diffusa se si considera che al 9/3/2019 le occorrenze del termine tra virgolette nelle pagine in italiano di Google ammontano a circa 30.600.

Rispetto alle molte forme già ricordate di cui l'italiano dispone (e a cui si possono aggiungere i sintagmi costituiti da nome + tecnico/tecnicistico/tecnologico), tecnicalità ha il vantaggio di essere più astratto e assumere i valori di 'dettaglio, elemento, termine tecnico' (e in questo caso è molto spesso usato al plurale), di 'processo, procedura tecnica, insieme delle procedure tecniche necessarie a un determinato scopo', di 'competenza tecnica' e anche in modo comprensivo di 'insieme di tutti gli aspetti tecnici riguardanti uno specifico settore'. In alcuni ambiti specialistici, come il diritto, l'economia, la medicina, la psicologia, la voce è impiegata in tutti o in molti di questi significati.

Vista la sua astrattezza risulta un termine particolarmente versatile e perciò utile da spendere nei media e in politica; d'altra parte può risultare in questi impieghi poco preciso, detenere un carico informativo inferiore rispetto a quello delle possibili alternative. Nel linguaggio politico e in quello giornalistico sembra prevalere il significato di 'dettaglio/aspetto/termine tecnico (e perciò supposto incomprensibile ai più)'. In questo particolare senso tende ad assumere una connotazione negativa o ironica, specialmente quando si voglia opporre l'incisività del decisionismo alla complessità delle realtà a cui si applica. E sembra essere questo l'unico uso recepito dalla lessicografia.

A tecnicalità è legato l'aggettivo tecnicale, non registrato dai dizionari (neppure da quelli che riportano il sostantivo), ma presente nella sezione Neologismi Treccani con questa citazione dal "Foglio" datata I7/II/2007: 
E cosi rivedo in flashback sequenze dell'autunno '94: ribaltoni, coup d'état, ministri massoncelli e berluscones che si acconciano col bavero immadonnato di Oscar, le lacrime ricomuniste di San Silvestro per il sì a un governucolo tecnicale, promesse di riforme, bicamerali, baffini d'acciaio, scalate Telecom... e wow! mi sovviene l'eterno ritorno degli uguali.

Troviamo attestazioni meno recenti (risalenti al 2002 e al 2005) nella "Repubblica" - nessuna sulla "Stampa" e sul "Corriere" - in testi di Stefano Bartezzaghi dedicati a specifiche parole; a Bartezzaghi dobbiamo anche l'unica altra testimonianza (del 2008) presente sul quotidiano. Riportiamo quella che ci sembra più interessante:

Chi vuole affermare che le tecniche sono neutrali le chiama technicalities: un concetto ancora peggiore della parola che lo esprime. Diffidare della neutralità delle tecniche è un'ottima misura: lo si vede, una volta di più, nelle modalità del voto segreto alla Camera, dove i deputati devono infilare una mano in un pertugio dello scanno e pigiare uno dei tre pulsanti nascosti che stanno per voto favorevole, sfavorevole, astensione. Mera tecnicalità? Ma quando mai. Un franco tiratore deve usare l'intera mano per nascondere il suo voto. Se invece che la mano si infila un solo dito, ma proprio quello - è possibile solo il voto favorevole. [...] Questo dito è l'anulare: il dito dorato della fede e del coniugio, degli obblighi contratti in privato e dichiarati in pubblico. Poteva la tecnica essere meno neutrale e "tecnicale" di cosi? Il voto segreto: un voto da anulare (Anulare, $\mathrm{i} 3 / \mathrm{IO} / 2005)$.

Il termine può essere un adattamento dell'inglese technical, ma, almeno in queste testimonianze pare evidente il rapporto diretto con tecnicalità intesa in senso negativo. Non sarà un caso che 2002 e 2005 sono gli anni in cui si registrano i primi picchi nell'uso di tecnicalitá sui quotidiani.

Per quel che riguarda la rete, in cui è difficile dare una stima attendibile della frequenza (delle r.550 occorrenze virtuali di "tecnicale" o "tecnicali" in italiano, quelle pertinenti sono pochissime). Di visione tecnicale (e di logica tecnicale) si parla nell'ambito della psicologia clinica (cfr. Claudia Venuleo, Colloquiando (sul)le organizzazioni, "Scritti di Gruppo" n. 2, luglio 2007, pp. 85-Io8: 95, IO2) e in altri scritti dello stesso ambito disciplinare.

Questa prospettiva è illustrata in Renzo Carli, Massimo Grasso, Rosa Maria Paniccia, La formazione alla psicologia clinica. Pensare emozioni, Franco Angeli, 2007. In particolare a p. I26 leggiamo:

Analizzando il caso di tirocinio nella scuola si cominciano a cogliere differenze tra tecnicalità e intervento fondato sull'analisi della domanda, differenze di ordine metodologico che rimandano a differenze entro i percorsi e i modelli della formazione. [...] Coerentemente con questi presupposti, la formazione ad una prassi tecnicale non implica una competenza a leggere il contesto.

E tecnicale, in opposizione all'aggettivo relazionale, si trova in Francesco Calamo Specchia, Comunicazione profonda in sanitá: senso, verità, desiderio, Maggioli editore, 20II, p.II5:

È dunque assolutamente sconcertante che la filosofia relazionale di questi processi e di questi approcci non trovi ancora spazio sufficiente in una Sanità pubblica [...] e che l'organizzazione che per la sua natura meritoria dovrebbe essere relazionale più d'ogni altra [...] si mantenga invece spesso chiusa, autocentrata, tecnicale, rigida...

Si tratta di un adattamento di technical usato in testi inglesi di psicologia in rapporto a relational e situational.

Queste, insieme a un'attestazione in Effetto domino di Diego Pierini (Lampi di stampa, 2orI), sono le uniche attestazioni pertinenti dell'aggettivo che troviamo nel corpus di Google Libri per il nostro secolo. Ne abbiamo poi rintracciate due in testi del secolo scorso: 
E sono snervato, perché il Convegno fu fiacco (a parte la giornata di ieri, teatrale o tecnicale) e bisogna tenerlo su colla frusta (Filippo Turati, AnnaKuliscioff,Carteggio: 1910-1914, a cura di Alessandro Schiavi e Franco Pedone, G. Einaudi, I977, vol. III, p. 165).

L'etica tecnicale - mi si consenta questo neologismo che vuole evitare stilemi sbrigativamente sprezzanti come «tecnicistico» - è una cosa altamente apprezzabile (Luciano Cafagna, Dualismo e sviluppo nella storia d'Italia, Marsilio, 1989 p. xvi).

Sono testimonianze molto diverse da quelle riscontrate sulla stampa, per lo più riferibili ad ambiti specialistici; in esse l'aggettivo,per quanto non sempre del tutto neutro, non ha certo la carica dispregiativa e ironica riscontrate nelle attestazioni sulla "Repubblica" e sul "Foglio".

Sia per tecnicale che per tecnicalità dunque occorre distinguere i due piani di impiego: quello specialistico in cui il valore semantico è più esteso e diversificato e quello giornalistico in cui predomina l'aspetto connotativo.

\section{Cita come:}

Matilde Paoli, La tecnicalità è un (prestito di) lusso?, "Italiano digitale", 2019, IX, 2019/2 (aprilegiugno)

DOI: $10.35948 / 2532-9006 / 2020.3106$

\section{Copyright 2019 Accademia della Crusca}

Pubblicato con licenza creative commons CC BY-NC-ND 